\title{
Stylistic Grammars in Language Translation
}

\author{
Chrysanne DiMARCO and Graeme HIRST \\ Department of Computer Science \\ University of Toronto \\ Toronto, Canada M5S 1A4
}

\begin{abstract}
We are developing stylistic grammars to provide the basis for a French and English stylistic parser. Our stylistic grammar is a branching stratificational model, built upon a foundation dealing with lexical, syntactic, and semantic stylistic realizations. Its central level uses a vocabulary of constituent stylistic elements common to both English and French, while the top level correlates stylistic goals, such as clarity and concreteness, with patterns of these elements.

Overall, we are implementing a computational schema of stylistics in French-to-English translation. We believe that the incorporation of stylistic analysis into machine translation systems will significantly reduce the current reliance on human post-editing and improve the quality of the systems' output.
\end{abstract}

\section{Introduction}

Current machine translation (MT) systems deal only superficially, if at all, with the translation of style. At best, MT output is syntactically correct but strictly neutral in tone. The expressive effects contained in the source text, together with the associated meaning, are lost.

The translation of style involves two complementary and sometimes conflicting aims:

- Producing a style appropriate to the particular target language, while

- Preserving the original author's stylistic intent.

These aims require an understanding of the internal stylistics of both the source and target languages as well as the comparative stylistics of the language pair.

Our focus is the development of stylistic grammars that will provide the basis for an English and French stylistic parser. The ultimate aim is the design and implementation of a computational schema of stylistics in French to English translation that will act as a post-editor to modify the output of a basic MT system to achieve the abovementioned complementary aims.

We believe that MT is an excellent vehicle for research into stylistics and that the incorporation of stylistic analysis into MT systems will significantly reduce the current reliance on human post-editing and will improve the quality of the system output.

\section{A Definition of Style for Machine Translation}

Our approach to a definition of style is non-literary, groupbased, and, most important, goal-directed. A "groupbased" approach emphasizes the stylistic standards shared by a body of writers, rather than the characteristics of an individual author. Examples of group styles can be found in newspaper reporting or scientific writing. By "goal-directed", we mean that we seek to explicitly correlate specific goals of style such as clarity, informality, or abstraction with particular synțactic structures and lexical choices.

Why focus on group-based style? For MT, where we expect to deal with large amounts of similar types of text, the analysis of group style is of more interest than the idjosyncratic style of any one writer. However, group style can be subdivided into two major types, literary and functional (or pragmatic). Analysing literary group style, as in a work of fiction, for example, is too ambitious a task for MT. Functional group styles, on the other hand, are correlated with particular types of situations and are a more realistic objective.

To fulfill both of the complementary aims, we require a view of style that is goal-directed. A recent example with this view of stylistics is the PAULINE system described in Hovy [1987]. PAULINE generates text that conforms to given pragmatic and stylistic constraints; the system is goal-directed, able to correlate such stylistic goals as formality, simplicity, and respect with the characteristics of the text produced.

In French-to-English translation, as an example, we must account for the fact that French is generally more abstract, static, and precise while English tends to be concrete, dynamic, and more lenient towards vagueness (Vinay and Darbelnet [1958]). For the same text, therefore, the French source language may express an abstract style while the English target language must be more concrete in its lexical and syntactic choices. If we are to properly translate from French style to English style, then we must understand how syntactic structures and lexical choices correlate with particular stylistic goals or aspects, such as abstraction, concreteness, staticness, and dynamism. 


\section{A Computational Schema of Stylistics in Translation}

We have restricted our interest in style for $\mathrm{MT}$ purposes to concen ns that are pragmatic, group-based, and goaldirected. The next question is: how is this restricted definition to be reflected in a computational schema of stylistics in machine translation? The schema that we propose is outlined in Figure 1. The process of stylistic translation is language-independent but French-to-English translation is the particular instantiation that we have studied in detail and from which we will give examples.

In the first stage of the process, French internal stylistics is considered. For each stylistic translation unit, i.e., the curreat section of text within which the style remains constant, in the French source text, we determine its stylistic contest, i.e., a correlation of lexical choices and syntactic structures with a particular stylistic goal and a particular stylistic intensity.

In the second stage, we use knowledge of French-English comparative stylistics. For each stylistic translation unit in the French source text, given its stylistic con. text, we determine the appropriate English stylistic goal corresponding to the possibly different French style. $D e-$ fault rules of comparative stylistics are used but these can be broken if the situation warrants.

The third and final stage requires an understanding of English internal stylistics. For each stylistic translation unit in the unedited English translation, we edit lexical choices and syntactic structures to achieve an appropriate style that corresponds to the French source style, but is good English style as well.

The main feature of the schema is that the translation of style, according to our definition, requires three distinct bodies of stylistic knowledge: French internal stylistics, French--English comparative stylistics, and English internal stylistics. The internal stylistics of a language tells us how to choose syntactic structures and lexical itcms to express a particular aspect of style, such as clarity or economy. From comparative stylistics, we gain an understanding of when the target text should express a different style from the source text and when the styles should remain the same.

The need for three kinds of stylistic knowledge may not initially appear obvious. Why not just edit the output of a MT isystem to remove awkward syntax and achieve a more natural flow of words? The answer is that to preserve the author's stylistic intent, while meeting the stylistic demands of the target language, we must determine the author's specific stylistic goals and consider how these different uspects can be realized syntactically and lexically in the target language. Thus, we must analyse the original source text to make certain we understand the source language style before trying to translate.

To inplement the computational schema of stylistics in translation shown in Figure 1, knowledge about stylistics must be accumulated, created, and organized into a formal representation.

Our first task was the creation of a vocabulary of English stylistics. The definition of concepts and the attempt to organize them into a recognizable structure were necessary to understanding the problem of translating style. The development of the vocabulary proceeded in tandem with the collection and creation of stylistic rules.

A further complication in the codification of French, English, and comparative stylistics is the need to build different sets of rules for lexical choice, syntactic structure, and semantic structure. This knowledge goes to make up stylistic grammars -- formal representations of the stylistic rules. These grammars provide a systematic description of the lexical, syntactic, and semantic patterns that differentiate the various stylistic goals.

With these formal bodies of rules to analyse the original source and unedited target texts, the strategy to be used by a French and Englisl stylistic parser can be planned. Ultimately, all components will be integrated in a post-editor that will accomplish the actual iranstation of style.

\section{Stylistic Grammars}

As a first step towards constructing a gramuar of style, we defined the basic vocabulary shown in Table 1.

'The naive approach towards structuring an extencled vocabulary of stylistics would be to organize terms by syntactic category. For example, the concepts of detached adjoctival clause, piled-up adjectives, and adjectival phrose are all significant in stylistics. However, not ail terms associated with the same syntactic category necessarily share the same stylistic significance.

We have therefore taken a more stylistically relevant approach by introducing stylistic elements, which provide both a means of structuring the vocabulary and a link be. tween these vocabulary terms and stylistic goals. Furthermore, a single vocabulary of stylistic elements will be used to describe lexical, syntactic, and semantic realizations.

Style, we believe, is achieved through the effects inherent in individual components (absolute shape), the effccts created by the absolute position of components (whether, for example, the component is initial, medial, or final), and by relationships (relative shope) between the components of a stylistic constituent. These relationships fall into at least two basic types: balance and harmony, including the concordant and discordant elements in a constituent, and dominance, the number of contral shapes in a constituent.

Each type of relationship is expressed by stylistic alements. Table 2 shows samples. All the stylistic elements that we have defined, about twenty, have been recognized in our sample English data. Given these elements, we have a means of organizing the terms in our stylistic vocabulary: each term is associated with one or more stylistic elements of the various types. 


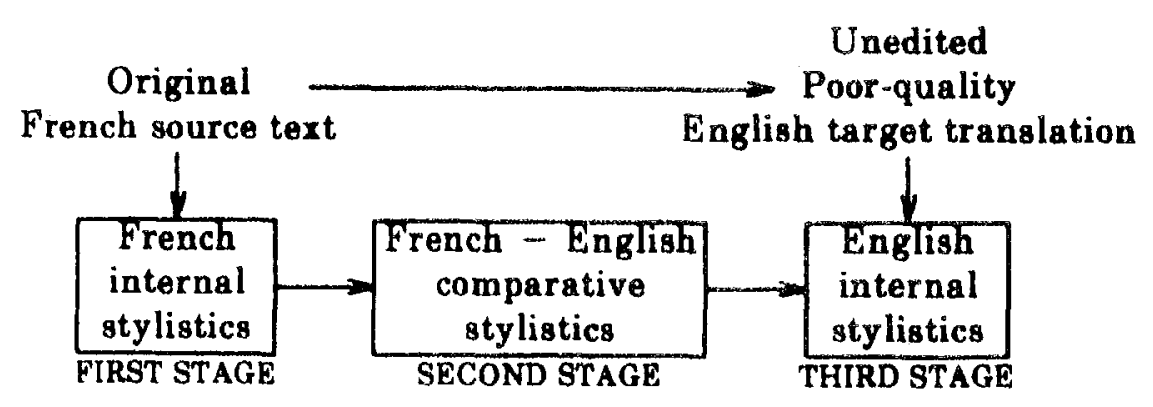

Figure 1: A Computational Schema of Stylistics in Machine Translation

\begin{tabular}{|l|l|}
\hline Stylistic constituent: & $\begin{array}{l}\text { A passage of text, associated with a particular stylistic goal, } \\
\text { within which the style remains constant, e.g., a sentence or paragraph. }\end{array}$ \\
\hline Stylistic component: & A part of a stylistic constituent, e.g., a phrase or clause. \\
\hline Stylistic shape: & $\begin{array}{l}\text { A stylistic component usually regarded as stylistically expressive, } \\
\text { i.e., having a particular stylistic effect. }\end{array}$ \\
\hline Stylistic texture: & $\begin{array}{l}\text { A stylistic constituent with an overall stylistic effect, } \\
\text { formed by particular types of relationships between its individual shapes. }\end{array}$ \\
\hline Stylistic equivalence class: & \begin{tabular}{l} 
A set of stylistic constructions having the same type of stylistic shape. \\
\hline Interval:
\end{tabular} \\
\hline Modulation: & $\begin{array}{l}\text { A shift within a stylistic constituent from one type of stylistic effect, e.g., concord, } \\
\text { to another, e.g., discord. }\end{array}$ \\
\hline
\end{tabular}

Table 1: Basic Vocabulary of Style

\begin{tabular}{|l|l|}
\hline Monopoise: & A syntactically complete stylistic texture with no disturbance in canonical order. \\
\hline Counterpoise: & $\begin{array}{l}\text { A stylistic texture containing an offset, a shape which perturbs the stylistic balance } \\
\text { by disturbing the canonical order. }\end{array}$ \\
\hline Homopoise: & A counterpoise in which the offset supports the overall stylistic balance. \\
\hline Polypoise: & A counterpoise in which the offset opposes the overall stylistic balance. \\
\hline \hline Concord: & $\begin{array}{l}\text { A stylistic shape expressing a unity of style, } \\
\text { agreement, accord, stability, and not requiring resolution. }\end{array}$ \\
\cline { 2 - 3 } & $\begin{array}{l}\text { A modulation with a narrow interval between two shapes, each expressing consonance. } \\
\text { Discord: } \\
\text { disagreement, contention, conflict, incongruity, and requiring resolution. }\end{array}$ \\
\cline { 2 - 3 } & $\begin{array}{l}\text { A modulation with a wide interval. } \\
\text { A stylistic texture in which the initial and terminal shapes belong to } \\
\text { the same stylistic equivalence class. }\end{array}$ \\
\hline Cycle: & $\begin{array}{l}\text { A stylistic texture in which two or more successive shapes belong to } \\
\text { the same equivalence class. }\end{array}$ \\
\hline Imitation: & A terminal modulation which moves from a stylistic discord to a relative concord. \\
\hline Resolution: & A terminal modulation which moves from a stylistic concord to a relative discord. \\
\hline Dissolition: & \begin{tabular}{l} 
A stylistic constituent having a stylistic shape but syntactically incomplete. \\
\hline \hline Aschematic:
\end{tabular} \\
\hline Monoschematic: & $\begin{array}{l}\text { A stylistic constituent with a single dominant shape and } \\
\text { no accompanying subordinate shapes. }\end{array}$ \\
\hline Diaschematic: & $\begin{array}{l}\text { A stylistic constituent in which the components are organized around } \\
\text { a single dominant shape. }\end{array}$ \\
\hline Polyschematic: & A stylistic constituent organized around two or more dominant shapes. \\
\hline
\end{tabular}

Table 2: Examples of Stylistic Elements 
Now, for our goal-directed stylistic grammar, we propose a branching stratificational model. As the foundation, we have three branches: lexical, syntactic, and semantic stylistic grammars, each with its own vocabulary of stylistic shapes and rules relating its type of shapes.

At the central level, we use a single vocabulary of constituent elements of style, as in Table 2, to build rules relating these clements to patterns of lexical, syntactic, and semantic stylistic shapes. This level is the unifying core of the overall stylistic grammar for we belicve that people also use the same principles, the same constituent elements, to express style both in English and French.

Finally, at the top level, we construct rules to correlate individual stylistic goals with patterns of stylistic elements. Together, these levels form a language-independent goaldirected stylistic grammar for language translation.

\subsection{A Syntactic Stylistic Grammar}

For each of the branches in our goal-clirected stylistic grammar, we must define a vocabulary of stylistic shapes and rules for puttiug these shapes together. However, a "stylistic shape" will be defined differently for the lexical, syntactic, and seraantic branches.

For the syntactic stylistic grammar, wo have built a catalogue of sentence components (syntactic stylistic shapes) organized into equivalence classes by stylistic ef$f e c t$, a quality inherent in a component which makes it a stylistic shape. But what, in syntactic terms, gives each component a particular stylistic effect? How should we define "stylistic shape" at the syntactic level?

We have adapted Quirk and Greenbaum's [1979] usc of syntactic integration as the basis for our definition of syntactic stylistic shape. They catalogue most advcrbials, some prepositional phrases, and some clauses as either axljunct, conjunct, or disjunct depending on the degree of inherent integration. We have chosen to base the syntactic stylistic effect of all sentence components on their characteristic integration. We have therefore expanded the catalogue to define a syntactic stylistic vocabulary classifying components as either adjunct (strongly integrating), conjunct (less integrating, but still having a connective effect), disjunct (neutral), or "antijunct" (having a disconnective effect).

We then adopted Crystal and Davy's [1969] "stylistic" grammar as the foundation for our syntactic stylistic grammar. Their grammar is built upon a vocabulary of stylistically significant syntactic components. It also recognizes the role of pre- and post-modification in stylistic effects, a feature we have expanded in our grammar, a portion of which is shown in Table 3.

Now, having a base syntactic stylistic grammar which builds sentences out of syntactic stylistic shapes defined by their characteristic integrating effect, we can construct, at the next level, a grammar which relates constituent clements of style to patterns of syntactic stylistic shapes. Syntactic examples for this level are given in Table 4.

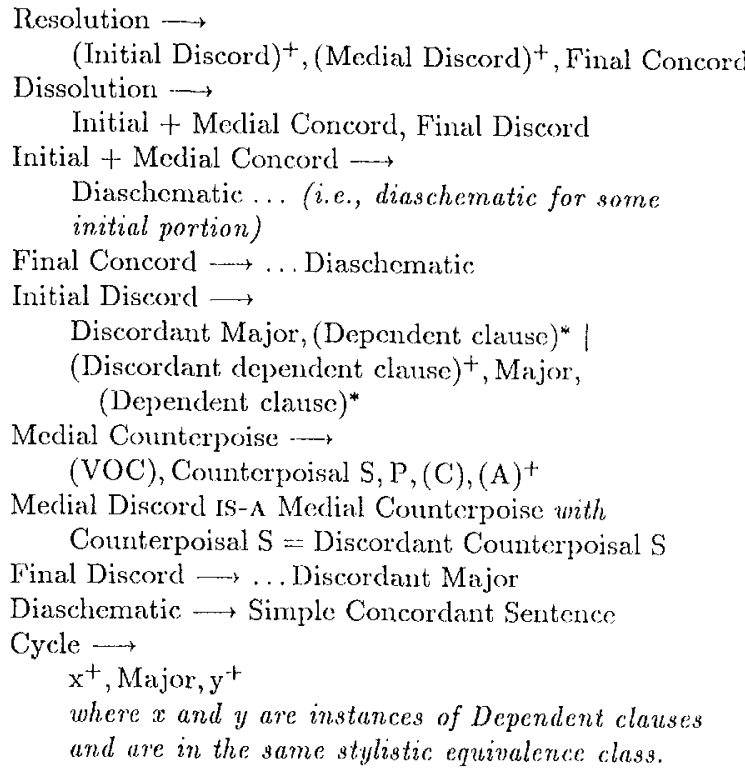

Table 4: Sample Constituent Flement Stylistic Grammar

Clarity $\longrightarrow$ Diaschematic / Resolution

Clarity not $\longrightarrow$ Initial Discord + Medial Discord

Concreteness $\longrightarrow$ Aschematic | Medial Counterpoise | Dissolution | Cycle

Table 5: Sample Grammar of Stylistic Goals

And, finally, at the top level, we define rules which correlate stylistic goals with patterns of constituent clements. Syntactic clarity and syntactic concreteness, for example, are correlated positively and negatively with the stylistic elements shown in Table 5.

Taken together, tables 3,4 and 5 provide a portion of our goal-directed stylistic grammar for English internal stylistics.

The use of this grammar can be illustrated by the analysis of the following two sanple sentences: they are diaschematic and thus express clarity. This first examplo illustrates imitative dependent clutses: This painter did his best to adjust to the tastes of the day, softening his line. and painting gracefully, converting to sfumato and casting about for art-lovers even as far away as in Spanish America [Manchester Guardian, February 14, 1988, p.15]. And, in this example, we see imitative postmodification: Silvia, a commanding woman in her 50's, a shrew falsely mellowed by religion, promptly organised prayer sessions on the lines of Tupperware meetings [adapted from Manch- 
Sentence $\longrightarrow$ Complete | Incomplete

Simple Concordant Sentence IS-A Sentence with only Complete $=$ Simple Concordant Complete

Complete $\longrightarrow$ (Dependent clause) ${ }^{*}$, Major, (Dependent clause)* | Minor

Simple Concordant Complete $\longrightarrow$ Simple Concordant Major,(Concordant dependent clause)*

Major $\rightarrow(\text { Conjunction })^{*},(\mathrm{~A})^{*},(\mathrm{C}),(\mathrm{VOC}), \mathrm{S}, \mathrm{P},(\mathrm{C}),(\mathrm{A})^{+}$

Simple Concordant Major $\longrightarrow$

(Conjunction)*, Simple Concordant S, P, (Simple Concordant C), (Concordant A)

Discordant Major $\longrightarrow$

(Conjunction) $)^{*},(\text { Discordant } \mathrm{A})^{+},(\mathrm{C}),(\mathrm{VOC}), \mathrm{S}, \mathrm{P},(\mathrm{C}),(\mathrm{A})^{+}$

(Conjunction)* ${ }^{*}(\mathrm{~A})^{*},(\mathrm{C}),(\mathrm{VOC}),(\text { AuxVerb) })^{+},(\mathrm{C}), \mathrm{S}, \mathrm{P},(\mathrm{C}),(\mathrm{A})^{+}$

(Conjunction)* ${ }^{*},(\mathrm{~A})^{*},(\mathrm{C}),(\mathrm{VOC}), \mathrm{P}, \mathrm{S},(\mathrm{C}),(\mathrm{A})^{+}$

Discordant $\mathrm{A} \longrightarrow$ Disjunct or Antijunct adverb | Disjunct or Antijunct adverbial construction |

Disjunct or Antijunct dependent [adverbial] clause

$\mathrm{S} \longrightarrow$ Nominal group | Pronoun | Dependent [noun] clause

Simple Concordant S IS-A S with only ((Nominal group = Simple Concordant Nominal group) | Pronoun)

Counterpoisal S IS-A S with only Nominal group = Counterpoisal Nominal group

Discordant Counterpoisal S IS-A Counterpoisal S with

Nominal group $=$ Discordant Counterpoisal Nominal group

Nominal group $\longrightarrow$ (Premodification ${ }_{\mathfrak{n}}$ ) Noun (Postmodification)* $\mid$ Pronoun

Simple Concordant Nominal group IS-A Nominal group with

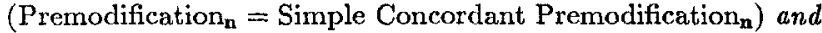

(Postmodification $=($ Simple Concordant Postmodification $)$

Counterpoisal Nominal group Is-A Nominal group with

Postmodification $=(\text { Counterpoisal Postmodification })^{+}$

Discordant Counterpoisal Nominal group Is-A Counterpoisal Nominal group with

Postmodification $=(\text { Discordant Counterpoisal Postmodification })^{+}$

Simple Concordant Premodification IS-A Premodification with only Adjunct or Conjunct Premodification

Postmodification $_{\mathbf{n}} \longrightarrow$

Adjunct Postmodification $_{\mathbf{n}} \longrightarrow$ Dependent [relative] clause | Nominal relative clause |

Adjunct dependent [adverbial] clause

Conjunct Postmodification $\mathbf{n}_{\mathbf{n}} \longrightarrow$ Non-finite construction | Conjunct dependent [adverbial] clause |

Nominal "that" clause | Preposition* + Nominal group

Disjunct Postmodification $_{\mathbf{n}} \longrightarrow$ Verbless clause | Disjunct dependent [adverbial] clause

Antijunct Postmodification $\mathbf{n}_{\mathbf{n}} \longrightarrow$ Adjective

Simple Concordant Postmodification $\longrightarrow$

Adjunct or Conjunct Postmodification (Imitative Postmodification) | Imitative Postmodification

Imitative Postmodification $\longrightarrow$

$\mathrm{x}$, (Conjunction), $\mathrm{y}$, (Conjunction), $(\mathrm{z})$ where $x, y, z$ are instances of Postmodification and

are not instances of Antijunct Postmodification and are in the same stylistic equivalence class.

Counterpoisal Postmodification IS-A Postmodification with all except

Conjunct Postmodification $\neq$ Preposition* + Nominal group

Discordant Counterpoisal Postmodification IS-A Counterpoisal Postmodification with only Antijunct Postmodification

Concordant dependent clause $\longrightarrow$ Adjunct or Conjunct dependent clause $\mid$ Imitative dependent clauses

Discordant dependent clause $\longrightarrow$ Disjunct dependent clause | Antijunct dependent clause

Adjunct dependent [adverbial] clause $\longrightarrow$

time-adverbial-clause $\longrightarrow$ time-adverb, Major

place-adverbial-clause $\longrightarrow$ place-adverb, Major

purpose-adverbial-clause $\longrightarrow$ purpose-adverb, Major

time-adverb $\longrightarrow$ since, usually, before, after, until, when, ...

place-adverb $\longrightarrow$ where, wherever, ...

purpose-adverb $\longrightarrow$ in order to, so as to, ...

Table 3: Sample Syntactic Stylistic Grammar with Modified Crystal and Davy Notation 
ester Guardian, February 7, 1988, p.16]. Note that our stylistic gyammar also gives patterns of stylistic elements which tend to oppose a certain stylistic goal. For example, an initial discord, a disjunct dependent [non-finite] clause, followed by a medial discord, an antijunct postmodification, a postposed adjective, tends to suppress clarity in the following sentence: To illustrate the benefits of assertiveness, politicians, eloquent, captire our interest.

In these next two sentences, we see an effect of syntactic concreteness, an attempt to express the immediacy of the world in the syntactic structure. The first sentence contains a medial counterpoise: Now demons, whatever else they nuay be [nominal relative clause], are full of interest. The second example contains a dissolution: And the rain descended [simple concordant major] and the floods came, and the winds blew, and beat upon the house; and it fell: [concordant dependent clause due to the imitation] and great was the fall of it [discordant major due to the inversion of the subject and predicate] [Matthew, 7:27].

We can now begin to see how our vocabulary of constituent elements, the central level of our stylistic grammar, will apply to the development of a semantic stylistic grammar. In the following two sentences, for example, we see semantic counterpoise, the main idea interrupted by a secondary one: The university attended by the Prime Minister, one of the finest law schools in the country, is the alma mater of many prominent politicians. Compare this with: The university attended by the Prime Minister, a set of buildings with the architectural charm of a prison, is the alma mater of many prominent politicians. In the first case, the offet supports the main idea so that there is an overall effect of stylistic concord but, in the second example, the intruding phrase is semantically and stylistically discordant.

In more subtle ways, we can correlate stylistic elements with patterns of semantic structure. In the following sentences, note the difference in order of components between $I l$ a regardé dans le jardin par la porte ouverte and its English translation, He gazed out of the open door into the garden. In French, the result comes first, then the means, while English follows the order of the images, like a film of the action [Vinay and Darbelnet 1958]. We would say that that are three types of semantic stylistic shapes composing the sentence: he gazed, out of the open door, and into the garden. In the English sentence, the modulations between shapes seem small, so that there is a gradual unfolding of images, an effect of concreteness, while the French structure contains a pattern of modulations characteristic of abstraction.

So far, we have put forward stylistic grammars as the formal representation of English and French internal stylistics. This approach has led us to organize a stylistic vocabulary describing sentence components in terms of stylistic shapes and then to define rules relating stylistic elements to patterns of these shapes. The next step, associating stylistic goals with stylistic elements allows us, in effect, to correlate syntactic structures with these goals.
With an explicit correlation between syntactic grammar rules, stylistic grammar rules, and stylistic goals, we can now propose a strategy for the English and French stylistic parser that we will construct. The syntactic rules do the driving but partial stylistic decisions can affect the direction of the syntactic parse. That is, once a stylistic rule has been partially recognized, we can narrow the search space for constructs to complete the rule. As the syntactic rules analyse sequences of syntactic structures, these structures can then be recognized as sequences of stylistic elements, the components of stylistic rules. In tandem, the stylistic rules will pick up a sequence of stylistic elements and associate it with a stylistic goal. The idea of tandem rules in language analysis is developed by Hirst [1987]. This approach to integrating syntax and semuntics has been adapted to syntax and stylistics.

\section{Summary}

- Our recent work has been the construction of a goaldirected stylistic grammar for English internal stylistics. Our current focus is an extension of the grammar for French internal stylistics. As well, we arc using a single vocabulary of constituent stylistic elements as the guiding principle in the development of a semantic stylistic grammar, the second branch of our stratificational model.

Our use of stylistic grammars has allowed us to propose a strategy for the French and English stylistic parser which will eventually form paris of an editor to accomplish the actual translation of style.

\section{Acknowledgements}

For discussion, helpful ideas, and comments on earlier drafts, we are grateful to Brian Fitch, Diane Horton, and Fduard Hovy. This work was supported in part by a grant to the second author by the Natural Sciences and Engineering Research Council of Canada.

\section{References}

[1] Crystal, David and Davy, Derek. Investigating English Style. Longmans, Green and Co. Limited, 1969.

[2] Hirst, Graeme. Semantic interpretation and the resolution of ambiguity (Studies in natural language processing). Cambridge University Press, 1987.

[3] Hovy, Eduard Hendrik. Generating Natural Language Under Pragmatic Constraints. PhD thesis, Department of Computer Science, Yale University, March 1987.

[4] Quirk, Randolph, and Greenbaum, Sidney. A University Grammar of English, Longman Group Limited, 1979.

[5] Vinay, J.-P. and Darbelnet, J. Stylistique comparée du français et de l'anglais. Didier, Paris, 1958. 